\title{
Regularity of Minimizers of Integrals of the Calculus of Variations with Non Standard Growth Conditions
}

\author{
PaOlo Marcellini
}

\author{
Communicated by E. GiUstI
}

\section{Introduction and statements of the main results}

In this paper we will consider integrals of the calculus of variations of the type

$$
\int_{\Omega} f(D u(x)) d x
$$

where $f$ is a function of class $C^{2}\left(\mathbb{R}^{n}\right), \Omega$ is a bounded open set of $\mathbb{R}^{n}$ ( $n \geqq 2$ ), $u: \Omega \rightarrow \mathbb{R}$ is a scalar function of the Sobolev space $H^{1, p}(\Omega)$ for some $p>1$, and $D u: \Omega \rightarrow \mathbb{R}^{n}$ is the gradient of $u$.

We say that a function $u$ is a minimizer for the integral (1.1) if

$$
\int_{\Omega} f(D u) d x \leqq \int_{\Omega} f(D u+D \varphi) d x \quad \forall \varphi \in C_{0}^{1}(\Omega) .
$$

In the following we will be more precise about the class of functions in which to look for a minimizer.

The existence of a minimizer in the class of Sobolev functions $H^{1, p}(\Omega)$, with a fixed boundary value, can be proved under the assumption that $f$ is a strictly convex function satisfying

$$
m|\xi|^{p} \leqq f(\xi) \leqq M\left(1+|\xi|^{q}\right) \quad \forall \xi \in \mathbb{R}^{n},
$$

for $M \geqq m>0$ and $1<p \leqq q$. The minimizer exists in $H^{1, p}(\Omega)$ independently of the assumption in the right hand side of (1.3). In particular, the condition $p=q$ is not necessary in the existence theory.

On the contrary, the assumption (1.3) with $p=q$ has been considered crucial in the regularity theory. For example, GiaquinTA \& GiUsTI [6] proved that, under the only assumption (1.3) with $p=q>1$, every minimizer of the integral (1.1) is locally Hölder continuous in $\Omega$. More regularity on $u$ (up to $C^{\infty}$-regularity and analiticity) has been obtained under controllated growth conditions on the second derivatives of $f$, for example of the type

$$
m|\lambda|^{2} \leqq \sum_{i j} f_{\xi_{i} \xi_{j}}(\xi) \lambda_{i} \lambda_{j} \leqq M|\lambda|^{2} \quad \forall \xi, \lambda \in \mathbb{R}^{n},
$$


where $M \geqq m>0$ (see for example LADYZHENSKAYA \& URAL' TSEVA (Section 6 of Chapter 5 of [9]), Sections 1.10 and 1.11 by MORREY [13], GiUSTI (Section 8 of Chapter 5 of [8])). Note that (1.4) implies that (1.3) holds (with different constants $m, M$ and up to a linear function) with $p=q=2$.

Thus, for example, the following integral in three dimensions

$$
\int_{\Omega}\left(u_{x_{1}}^{2}+u_{x_{2}}^{2}+u_{x_{3}}^{2}+u_{x_{3}}^{4}\right) d x
$$

or, more generally, an integral of the type (1.1) with $f=f\left(\xi_{1}, \xi_{2}, \ldots, \xi_{n}\right)$ polynomial of different growth in the $\xi_{i}$, has minimizers in $H^{1,2}(\Omega)$ but, up to now, it was not known whether they were of class $C^{\infty}(\Omega)$, or even of class $C^{0}(\Omega)$.

Recently the interest on studying problems of the calculus of variations with $p<q$ has been pointed out. We cite for example [11], where a problem related to nonlinear elasticity is considered, and ACERBI \& FUSCo [1], who proved a $C^{1, *}$ partial regularity theorem for minimizers of integrals (1.1) with integrands $f$ twice continuously differentiable, without growth conditions on the second derivatives of $f$, although $f$ essentially behaves like in (1.3) with $p=q$. Both papers deal with vector valued functions $u: \Omega \rightarrow \mathbb{R}^{N}(N \geqq 1)$ and quasiconvex functions $f$.

Is the condition $p=q$ in (1.3) really necessary for the regularity of minimizers?

The aim of this paper is to show that the answer is no. In fact we propose an approach to the (everywhere) local regularity in the scalar case under non-standard growth conditions on $f$ and on the second derivatives of $f$. We will prove the following theorems $\mathrm{A}, \mathrm{B}, \mathrm{C}$ :

Theorem A. Let $f$ be a function of class $C^{2}\left(\mathbb{R}^{n}\right)$ such that

$$
\begin{gathered}
m \sum_{j=1}^{n}\left|\xi_{j}\right|^{q_{j}} \leqq f(\xi) \leqq M\left(1+\sum_{j=1}^{n}\left|\xi_{j}\right|^{q_{j}}\right), \\
m|\lambda|^{2} \leqq \sum_{i, j=1}^{n} f_{\xi_{i} \xi_{j}}(\xi) \lambda_{i} \lambda_{j} \leqq M\left(1+\sum_{j=1}^{n}\left|\xi_{j}\right|^{a_{j}-2}\right)|\lambda|^{2},
\end{gathered}
$$

for every $\xi$ and $\lambda \in \mathbb{R}^{n}$, where $m$ and $M$ are positive constants and

$$
2 \leqq q_{j}<\frac{2 n}{n-2} \quad \forall j=1,2, \ldots, n
$$

$\left(2 \leqq q_{j}, \forall j\right.$, if $\left.n=2\right)$. Let $u$ be a minimizer of the integral (1.1). Then $u \in$ $H_{\mathrm{loc}}^{1, \infty}(\Omega)$ and for every $\Omega^{\prime} \subset \subset \Omega$ there is an increasing function $\psi:[0,+\infty) \rightarrow$ $[0,+\infty)$ such that

$$
\|D u\|_{L^{\infty}\left(\Omega^{\prime}\right)} \leqq \psi\left(\sum_{j=1}^{n}\left\|u_{x_{j}}\right\|_{L^{q_{j}}}\right)
$$

Theorem B. Let $f$ be a function of class $C^{2}\left(\mathbb{R}^{n}\right)$ such that

$$
m|\lambda|^{2} \leqq \sum_{i, j=1}^{n} f_{\xi_{i} \xi_{j}}(\xi) \lambda_{i} \lambda_{j} \leqq M\left(1+|\xi|^{q-2}\right)|\lambda|^{2},
$$


for every $\xi, \lambda \in \mathbb{R}^{n}$, with $m$ and $M$ positive constants and

$$
2 \leqq q<\frac{2 n}{n-2}
$$

( $2 \leqq q$, if $n=2)$. Let $u \in H_{\mathrm{loc}}^{1, q}(\Omega)$ be a minimizer of the integral (1.1). Then $u \in H_{\mathrm{loc}}^{1, \infty}(\Omega)$ and, for $\Omega^{\prime \prime} \subset \subset \Omega^{\prime} \subset \subset \Omega$, there is an increasing function $\psi:[0,+\infty)$ $\rightarrow[0,+\infty)$ such that

$$
\|D u\|_{L^{\infty}\left(\Omega^{\prime \prime}\right)} \leqq \psi\left(\|D u\|_{L^{q}\left(\Omega^{\prime}\right)}\right)
$$

Theorem C. Let f be a function of class $C^{2}\left(\mathbb{R}^{n}\right)$ satisfying (1.9) with $0<m \leqq M$ and

$$
2 \leqq q \leqq \frac{2 n}{n-1} \quad \text { if } \quad n>3
$$

while $2 \leqq q<3$, if $n=2$ or $n=3$. Let $u \in H^{1,2}(\Omega)$ be a minimizer of the integral (1.1) (the integral is well defined on $H^{1, q}(\Omega)$ and it is extended (see Section 6 ) "by semicontinuity" to $\left.H^{1,2}(\Omega)\right)$. Then $u \in H_{\mathrm{loc}}^{\mathrm{1}, q}(\Omega)$ and thus, by Theorem $B$, $u \in H_{\text {loc }}^{1, \infty}(\Omega)$. Moreover, for $\Omega^{\prime} \subset \subset \Omega$, there are constants $c>0$ and $\alpha \in(1,2]$ such that

$$
\|D u\|_{L^{\left.q_{(} \Omega^{\prime}\right)}} \leqq c\|1+|D u|\|_{L^{2}(\Omega)}^{\frac{q-2+\alpha}{*}} .
$$

We emphasize the fact that, in Theorem $C$, the integral (1.1) needs to be extended from $H^{1, q}(\Omega)$ to $H^{1,2}(\Omega)$. This problem has been studied in [11]. Here we show (see Section 6) how to use the extension to get regularity.

Theorems A, B, C are proved respectively in Sections $4,5,6$. In Section 7 we propose the (standard) use of the $H^{1, \infty}$ regularity to get the $C^{\infty}$ regularity. For example, by Theorem A, every minimizer of the integral (1.5) is of class $C^{\infty}(\Omega)$.

In Section 7 we discuss also the fact that integrals of the types considered in Theorems A, B, C may have discontinuous minimizers if the exponent $q$ is large enough with respect to $n$.

Let us briefly mention a technical difficulty arising in the proof of Theorem A: it seems necessary to consider separately each component of the gradient $D u$ to prove its pointwise local boundness. The prove is divided in $n$ steps. We procede first to estimate the partial derivative $u_{x_{s}}$ of "maximal growth" (see Section 4); then we use the fact that $u_{x_{s}} \in L_{\mathrm{loc}}^{\infty}(\Omega)$ to estimate the other partial derivatives, and so on.

Apart from this and some other difficulties (like lack of homogeneity in the estimates), we use a method of iteration that can be found in the literature in similar contexts. We refer to the method of Moser [14] to prove the local boundness of solutions of linear elliptic equations. We refer also to LADYZHENSKAYA \& URAL'TSEVA ([9], Section 3 of Chapter 4) and to GiUstr ([8], Section 8 of Chapter 5). In particular the book by GIUSTI is full of suggestions toward the results presented here. 


\section{Euler's equation}

The aim of this section is to derive Euler's equation in weak form, in particular to specify the function spaces in which to consider the minimizers and the test functions.

Lemma 2.1. Let $f=f(\xi)$ be a function defined on $\mathbb{R}^{n}$ such that

$$
|f(\xi)| \leqq M\left(1+\sum_{j=1}^{n}\left|\xi_{j}\right|^{q_{j}}\right) \quad \forall \xi \equiv\left(\xi_{j}\right) \in \mathbb{R}^{n},
$$

where $M>0$ and $q_{j} \geqq 1$ for $j=1,2, \ldots, n$. Let $\xi_{i}$ be a component of the vector $\xi$ and let us assume that $f$ is convex with respect to $\xi_{i}$. Then the partial derivative $f_{\xi_{i}}$ (which exists almost everywhere) satisfies

$$
\left|f_{\xi_{i}}(\xi)\right| \leqq c_{1}\left(1+\sum_{j=1}^{n}\left|\xi_{j}\right|^{q_{j}}\right)^{\left(1-\frac{1}{q_{i}}\right)} \quad \forall \xi \in \mathbb{R}^{n},
$$

for some positive constant $c_{1}$. In particular, if $q_{j}=q$ for every $j=1,2, \ldots, n$, then there is a $c_{2}>0$ such that

$$
\left|f_{\xi_{i}}(\xi)\right| \leqq c_{2}\left(1+|\xi|^{q-1}\right) \quad \forall \xi \in \mathbb{R}^{n} .
$$

Proof. We follow the method of [10] (Step 2 of Section 2). With abuse of notation let us denote by $f\left(\xi_{i}\right)$ the function $f$ when only the component $\xi_{i}$ of the vector $\xi$ varies. By the convexity of $f\left(\xi_{i}\right)$, and by the assumption (2.1), for every $h>0$ we have

$$
\begin{aligned}
f_{\xi_{i}} & \lessgtr \frac{f\left(\xi_{i} \pm h\right)-f\left(\xi_{i}\right)}{ \pm h} \\
& \lessgtr c_{3} \frac{1+h^{q_{i}}+\sum_{j=1}^{n}\left|\xi_{j}\right|^{q_{j}}}{ \pm h} .
\end{aligned}
$$

For $h=\left(1+\sum_{j=1}^{n}\left|\xi_{j}\right|^{q_{j}}\right)^{1 / q_{i}}$ we obtain

$$
\begin{aligned}
\left|f_{\xi_{i}}\right| & \leqq 2 c_{3}\left(1+\sum_{j=1}^{n}\left|\xi_{j}\right|^{q_{j}}\right) \cdot\left(1+\sum_{j=1}^{n}\left|\xi_{j}\right|^{q_{j}}\right)^{-1 / q_{i}} \\
& =2 c_{3}\left(1+\sum_{j=1}^{n}\left|\xi_{j}\right|^{q_{j}}\right)^{1-1 / q_{i}}
\end{aligned}
$$

Lemma 2.2. Let $f$ be a convex function of class $C^{1}\left(\mathbb{R}^{n}\right)$ satisfying (2.1) with $q_{j} \geqq 1$ for $j=1,2, \ldots, n$. Let $u \in H^{1,1}(\Omega)$ be a minimizer of the integral (1.1) such that

$$
u_{x_{j}} \in L^{q_{j}} \quad \forall j=1,2, \ldots, n
$$


Then, for every $i=1,2, \ldots, n$,

$$
f_{\xi_{i}}(D u) \in L^{q_{i}^{\prime}} \quad \text { with } \quad \frac{1}{q_{i}^{\prime}}+\frac{1}{q_{i}}=1,
$$

and $u$ satisfies the Euler equation

$$
\int_{\Omega} \sum_{i=1}^{n} f_{\xi_{i}}(D u) \varphi_{x_{i}} d x=0 \quad \forall \varphi \in H_{0}^{1,1}(\Omega): \varphi_{x_{i}} \in L^{q_{i}}, \quad \forall i .
$$

Proof. By (2.2) we have

$$
\left|f_{\xi_{i}}(D u)\right| \leqq c_{4}\left(1+\sum_{j=1}^{n}\left|u_{x_{j}}\right|^{q_{j}}\right)^{\frac{q_{i}-1}{q_{i}}} .
$$

Since $u_{x_{j}} \in L^{q_{j}}$ for $j=1,2, \ldots, n$, it follows that

$$
f_{\xi_{j}}(D u) \in L^{q_{i} /\left(q_{i}-1\right)}(\Omega)=L^{q_{i}^{\prime}}(\Omega) .
$$

Thus (2.5) holds. Now, with a standard argument, by using the mean value theorem, inequality (2.2) and Lebesgue's dominated convergence theorem, we obtain the Euler equation with $\varphi \in C_{0}^{1}(\Omega)$. Then, by (2.5), (2.6) holds for general $\varphi$ too.

\section{Some technical preliminaries}

For $\alpha \geqq 2$ and $k>0$ let us denote by $g_{x, k}(t)$ the function of the real variable $t:$

$$
g_{\alpha, k}(t)= \begin{cases}|t|^{\alpha-2} t & \text { if }|t| \leqq k \\ (\alpha-1) k^{\alpha-2}(t-k)+k^{\alpha-1} & \text { if } t>k \\ (\alpha-1) k^{\alpha-2}(t+k)-k^{\alpha-1} & \text { if } t<-k\end{cases}
$$

Lemma 3.1. For every $\alpha \geqq 2$ and $k>0, g_{x, k}$ is a Lipschitz-continuous function that satisfies

$$
g_{x, k}^{2}(t) \leqq \frac{2}{\alpha-1} g_{x, k}^{\prime}(t) \cdot|t|^{\alpha}
$$

Moreover the derivative $g_{\alpha, k}^{\prime}$ is increasing with respect to $k$ and

$$
\lim _{k \rightarrow+\infty} g_{x, k}^{\prime}(t)=(\alpha-1) \mid t^{\alpha-2}
$$

Proof. A computation gives

$$
g_{\alpha, k}^{\prime}(t)= \begin{cases}(\alpha-1)|t|^{\alpha-2} & \text { if }|t| \leqq k \\ (\alpha-1) k^{\alpha-2} & \text { if }|t|>k\end{cases}
$$


Thus $g_{\alpha, k}$ is a function of class $C^{1}(\mathbb{R})$ and its derivative is bounded with respect to $t$ (with a constant that depends on $k$ ). Moreover, for $|t| \leqq k$ we have

$$
\frac{g_{x, k}^{2}(t)}{g_{x, k}^{\prime}(t)}=\frac{|t|^{2 x-2}}{(\alpha-1) \mid t^{\mid \alpha-2}}=\frac{1}{\alpha-1} \mid t^{\alpha} .
$$

Thus (3.2) holds if $|t| \leqq k$. We will have (3.2) for $|t|>k$, too, if we prove it for $t>k$, since $g_{\alpha, k}$ is an odd function. For $t \geqq k$ we can compute the maximum of the ratio

$$
\frac{g_{\alpha, k}^{2}(t)}{g_{\alpha, k}^{\prime}(t) \cdot t^{\alpha}}=\frac{k^{\alpha-2}}{\alpha-1} \frac{[(\alpha-1) t-(\alpha-2) k]^{2}}{t} .
$$

If $\alpha=2$ the maximum is assumed for $t=k$ and is equal to $1 /(\alpha-1)$. By a computation we can see that, if $\alpha>2$, then the maximum of the right side is assumed for $t=k \alpha /(\alpha-1)$ and is equal to $4(1-1 / \alpha)^{\alpha} /(\alpha-1)$. Thus in every case we have

$$
g_{\alpha, k}^{2}(t) \leqq \frac{4}{\alpha-1}\left(1-\frac{1}{\alpha}\right)^{\alpha} g_{x, k}^{\prime} \cdot|t|^{\alpha} .
$$

This implies (3.2), since $(1-1 / \alpha)^{\alpha}<e^{-1}$. Now let us show that $g_{\alpha, h}^{\prime} \leqq g_{\alpha, k}^{\prime}$ if $h<k$ if $|t| \leqq h$ then $g_{x, h}^{\prime}=g_{\alpha, k}^{\prime}$; if $|t| \geqq k$ then

$$
g_{\alpha, h}^{\prime}=(\alpha-1) h^{\alpha-2} \leqq(\alpha-1) k^{\alpha-2}=g_{\alpha, k}^{\prime} ;
$$

finally, if $h<|t|<k$, then

$$
g_{x, h}^{\prime}(t)=(\alpha-1) h^{\alpha-2} \leqq(\alpha-1)|t|^{\alpha-2}=g_{\alpha, k}^{\prime}(t)
$$

This proves that $g_{\alpha, k}^{\prime}$ is increasing with respect to $k$. The relation (3.3) follows immediately from (3.4).

In this section we assume that $f$ is a function of class $C^{2}\left(\mathbb{R}^{n}\right)$ satisfying

$$
\begin{aligned}
& f(\xi) \leqq M\left(1+\sum_{j=1}^{n}\left|\xi_{j}\right|^{q_{j}}\right) \quad \forall \xi \in \mathbb{R}^{n}, \\
& \sum_{i j} f_{\xi_{i} \xi_{j}}(\xi) \lambda_{i} \lambda_{j} \geqq m|\lambda|^{2} \quad \forall \xi, \lambda \in \mathbb{R}^{n},
\end{aligned}
$$

for some constants $m$ and $M>0$ and $q_{j} \geq 2$ for every $j=1,2, \ldots, n$. Let us observe that, by (3.7), there is a constant $m^{\prime}$ such that $f(\xi) \geqq m^{\prime}|\xi|^{2}$ to within a linear function. fying

We will assume also that $u \in H^{1,2}(\Omega)$ is a minimizer of the integral (1.1) satis-

$$
u_{x_{j}} \in L^{q_{j}} \quad \forall i=1,2, \ldots, n,
$$

so that we can use the Euler equation (2.6).

Fixed $s \in\{1,2, \ldots, n\}$ we denote by $e_{s}$ the unit coordinate vector in the $x_{s}$ direction. Then we define the difference quotient in the direction $e_{s}$ by

$$
\Delta_{h} u(x)=\left(u\left(x+h e_{s}\right)-u(x)\right) / h
$$


for every $h \neq 0$. We do not denote explicitly the dependence on $s$. The function $\Delta_{h} u$ is defined in $\Omega_{h}=\{x \in \Omega: \operatorname{dist}(x, \partial \Omega)<h\}$ and, for every $j=1,2, \ldots, n$, we have

$$
\left(\Delta_{h} u\right)_{x_{j}}=\Delta_{h} u_{x_{j}} \in L^{q_{j}}\left(\Omega_{h}\right)
$$

For the properties of the difference quotient see for example Section 7.11 of [7], or Section 3 of Chapter 3 of [8], or Proposition IX.3 by [2].

Let $\eta \in C_{0}^{1}(\Omega), \eta \geqq 0$ in $\Omega$. For $|h|<(1 / 4)$ dist (supp $\eta, \partial \Omega$ ) let us define

$$
\varphi=\Delta_{-h}\left(\eta^{2} g_{x, k}\left(\Delta_{h} u\right)\right)
$$

where $g_{\alpha, k}$ (for $\alpha \geqq 2$ and $k>0$ ) is the function defined in (3.1). We have

$$
\varphi_{x_{i}}=\Delta_{-h}\left(\eta^{2} g_{\alpha, k}^{\prime}\left(\Delta_{h} u\right) \Delta_{h} u_{x_{i}}+2 \eta \eta_{x_{i}} g_{\alpha, k}\left(\Delta_{h} u\right)\right) \text {. }
$$

By (3.10) $\varphi_{x_{i}} \in L^{q_{i}}$ for every $i=1,2, \ldots, n$. We introduce $\varphi$ in the Euler equation (2.6). With standard computations we obtain

$$
\int_{\Omega} \sum_{i=1}^{n} \Delta_{h} f_{\xi_{i}}(D u)\left\langle\eta^{2} g_{\alpha, k}^{\prime}\left(\Delta_{h} u\right) \Delta_{h} u_{x_{i}}+2 \eta \eta_{x_{i}} g_{\alpha, k}\left(\Delta_{h} u\right)\right) d x=0
$$

Let us compute separately $\Delta_{h} f_{\xi_{i}}(D u)$ :

$$
\begin{aligned}
\Delta_{h} f_{\xi_{i}}(D u) & =\frac{1}{h} \int_{0}^{1} \frac{d}{d t} f_{\xi_{i}}\left(D u+t h \Delta_{h} D u\right) d t \\
& =\int_{0}^{1} \sum_{j} f_{\xi_{i} \xi_{j}}\left(D u+t h \Delta_{h} D u\right) \Delta_{h} u_{x_{j}} d t .
\end{aligned}
$$

Thus, by (3.2), the Cauchy-Schwarz inequality and the Hölder inequality, we obtain

$$
\begin{aligned}
& \int_{0}^{1} d t \int_{\Omega} \eta^{2} g_{\alpha, k}^{\prime}\left(\Delta_{h} u\right) \sum_{i, j} f_{\xi_{i} \xi_{j}}\left(D u+t h \Delta_{h} D u\right) \Delta_{h} u_{x_{i}} \Delta_{h} u_{x_{j}} d x \\
& =\int_{0}^{1} d t \int_{\Omega}-2 \eta g_{\alpha, k}\left(\Delta_{h} u\right) \sum_{i, j} f_{\xi_{i} \xi_{j}} \eta_{x_{i}} \Delta_{h} u_{x_{j}} d x \\
& \leqq \frac{2 \sqrt{2}}{\sqrt{\alpha-1}} \int_{\Omega} \eta\left\{g_{\alpha, k}^{\prime}\left(\Delta_{h} u\right)\left|\Delta_{h} u\right|^{\alpha}\right\}^{1 / 2}\left|\int_{0}^{1} \sum_{i, j} f_{\xi_{i} \xi_{j}} \eta_{x_{i}} \Delta_{h} u_{x_{j}} d t\right| d x \\
& \leqq \frac{2 \sqrt{2}}{\sqrt{\alpha-1}} \int_{S}\left\{\eta^{2} g_{\alpha, k}^{\prime}\left(\Delta_{h} u\right) \int_{0}^{1} \sum_{i, j} f_{\xi_{i} \xi_{j}} \Delta_{h} u_{x_{i}} \Delta_{h} u_{x_{j}} d t\right\}^{1 / 2} \\
& \left.\qquad \frac{2 \sqrt{2}}{\sqrt{\alpha-1}}\left\{\int_{0}^{1} d t \int_{\Omega} \eta^{2} g_{\alpha, k}^{\prime}\left(\Delta_{h} u\right) \sum_{i, j} f_{\xi_{i} \xi_{j}} \Delta_{h} u_{x_{i}}^{1} \int_{x_{h}} \sum_{i, j} f_{\xi_{i} \xi_{j}} \eta_{x_{i}} \eta_{x_{j}} d t\right\}^{1 / 2} d x\right\}^{1 / 2} \\
& \cdot\left\{\int_{0}^{1} d t \int_{\Omega}\left|\Delta_{h} u\right|^{\alpha} \sum_{i, j} f_{\xi_{i} \xi_{j}} \eta_{x_{i}} \eta_{x_{j}} d x\right\}^{1 / 2} \cdot
\end{aligned}
$$


We simplify both sides of the inequality and we obtain

$$
\begin{aligned}
& \int_{0}^{1} d t \int_{\Omega} \eta^{2} g_{\alpha, k}^{\prime}\left(\Delta_{h} u\right) \sum_{i, j} f_{\xi_{i} \xi_{j}} \Delta_{h} u_{x_{i}} \Delta_{h} u_{x_{j}} d x \\
& \leqq \frac{8}{\alpha-1} \int_{0}^{1} d t \int_{\Omega}\left|\Delta_{h} u\right|^{\alpha} \sum_{i, j} f_{\xi_{i} \xi_{j}} \eta_{x_{i}} \eta_{x_{j}} d x .
\end{aligned}
$$

First we use the assumption (3.7). Then, since the right hand side does not depend on $k$, we go to the limit as $k \rightarrow+\infty$. By Beppo Levi's monotone convergence theorem and by (3.3) we obtain

$$
\begin{aligned}
& \int_{\Omega} \eta^{2}\left|\Delta_{h} u\right|^{\alpha-2}\left|\Delta_{h} D u\right|^{2} d x \\
& \quad \leqq \frac{8}{m(\alpha-1)^{2}} \int_{0}^{1} d t \int_{\Omega}\left|\Delta_{h} u\right|^{\alpha} \sum_{i, j} f_{\xi_{i} \xi_{j}} \eta_{x_{i}} \eta_{x_{j}} d x .
\end{aligned}
$$

Let us recall that in the right hand side of (3.12) we have $f_{\xi_{i} \xi_{j}}=f_{\xi_{i} \xi_{j}}\left(D u+t h \Delta_{h} D u\right)$ and that (3.12) makes sense if the integral in the right-hand side is finite. Now let us compute separately the gradient of $\left|\Delta_{h} u\right|^{\alpha / 2}$; we obtain the estimate

$$
\left|D\left(\left|\Delta_{h} u\right|^{\alpha / 2}\right)\right|^{2} \leqq\left(\frac{\alpha}{2}\right)^{2}\left|\Delta_{h} u\right|^{\alpha-2}\left|\Delta_{h} D u\right|^{2} .
$$

From (3.12) and (3.13) we infer

$$
\int_{\Omega} \eta^{2}\left|D\left(\left|\Delta_{h} u\right|^{\alpha / 2}\right)\right|^{2} d x \leqq \frac{2 \alpha^{2}}{m(\alpha-1)^{2}} \int_{0}^{1} d t \int_{\Omega}\left|\Delta_{h} u\right|^{\alpha} \sum_{i, j} f_{\xi_{i} \xi_{j}} \eta_{x_{i}} \eta_{x_{j}} d x
$$

Now we use the inequality

$$
|D(\eta v)|^{2} \leqq 2\left(\eta^{2}|D v|^{2}+v^{2}|D \eta|^{2}\right) \quad \text { with } v=\left|\Delta_{h} u\right|^{\alpha / 2}
$$

Finally, we use Sobolev's inequality applied to the function $\eta v$, that has compact support in $\Omega$. The result that we have proved is stated in the following lemma.

Lemma 3.2. Let $f$ be a function of class $C^{2}\left(\mathbb{R}^{n}\right)$ satisfying (3.6), (3.7). Let $u$ be a minimizer of the integral (1.1) satisfying (3.8). There is a constant $c$ such that, for every $\alpha \geqq 2$ for which the integral on the right hand side is finite, we have

$$
\begin{aligned}
& \left(\int_{\Omega}\left(\eta\left|\Delta_{h} u\right|^{\alpha / 2}\right)^{2 *} d x\right)^{2 / 2^{*}} \\
& \quad \leqq c \int_{0}^{1} d t \int_{\Omega}\left|\Delta_{h} u\right|^{\alpha}\left(|D \eta|^{2}+\sum_{i, j} f_{\xi_{i} \xi_{j}}\left(D u+t h \Delta_{h} D u\right) \eta_{x_{i}} \eta_{x_{j}}\right) d x,
\end{aligned}
$$

where $2^{*}=2 n /(n-2)$ if $n>2$, and $2^{*}=$ any number greater than 1 if $n=2$. 


\section{Proof of Theorem A}

In this section we assume that $f$ is a function of class $C^{2}\left(\mathbb{R}^{n}\right)$ satisfying (1.6), (1.7) and (1.8). We will prove that every minimizer of the integral (1.1), in the class of functions satisfying (3.8), is locally Lipschitz-continuous in $\Omega$.

Let us interchange the names of the $x_{j}$-axes so that

$$
2 \leqq q_{1} \leqq q_{2} \leqq \ldots \leqq q_{n}<\frac{2 n}{n-2} .
$$

Let us denote by $B_{R}$ and $B_{Q}$ balls of center $x_{0}$ (we do not denote explicitly the dependence on $\left.x_{0}\right)$ and radii respectively $R, \varrho(R>\varrho>0)$, compactly contained in $\Omega$.

Lemma 4.1. Let us assume that (1.6), (1.7), (4.1) and (3.8) hold. Let $s \in\{1,2, \ldots, n\}$ and let us assume that the derivatives $u_{x_{s+1}}, u_{x_{s+2}}, \ldots, u_{x_{n}}$ belong to $L_{\mathrm{loc}}^{\infty}(\Omega)$. Then there exists a constant $c_{1}$ such that, if $u_{x_{s}} \in L^{\alpha q_{s} / 2}\left(B_{R}\right)$ for some $\alpha \geqq 2$, then (we consider the case $n>2$; if $n=2$ we have only to replace the expression $n /(n-2)$ by any real number $p>1) u_{x_{s}} \in L^{\alpha_{n} /(n-2)}\left(B_{e}\right)$ and

$$
\left(\int_{B_{Q}}\left|u_{x_{S}}\right|^{\frac{\alpha n}{n-2}} d x\right)^{\frac{n-2}{n}} \leqq \frac{c_{1}}{(R-\varrho)^{2}}\left(\int_{B_{R}}\left|u_{x_{S}}\right|^{\frac{\alpha q_{s}}{2}}\right)^{\frac{2}{q_{s}}} .
$$

Proof. By Lemma 3.2 and assumption (1.7) we have

$$
\begin{aligned}
& \left(\int_{\Omega}\left(\eta\left|\mathcal{A}_{h} u\right|^{\frac{\alpha}{2}}\right)^{\frac{2 n}{n-2}} d x\right)^{\frac{n-2}{n}} \\
& \quad \leqq c M \int_{0}^{1} d t \int_{\Omega}\left|\mathcal{A}_{h} u\right|^{\alpha}\left(2+\sum_{j=1}^{n}\left|u_{x_{j}}+t h \Delta_{h} u_{x_{j}}\right|^{\mid q_{j}-2}\right)|D \eta|^{2} d x .
\end{aligned}
$$

Let us take $\eta$ such that

$$
\eta \in C_{0}^{1}\left(B_{R}\right), \quad \eta \geqq 0, \quad \eta=1 \text { on } B_{\varrho}, \quad|D \eta| \leqq \frac{2}{R-\varrho} .
$$

We use the assumption that $u_{x_{s+1}}, \ldots, u_{x_{n}} \in L^{\infty}\left(B_{R}\right)$. We use also Hölder's inequality with exponents $q_{j} /\left(q_{j}-2\right)$ and $q_{j} / 2$. We get

$$
\begin{aligned}
& \left(\int_{B_{\varrho}}\left|\Delta_{h} u\right|^{\frac{\alpha_{n}}{n-2}} d x\right)^{\frac{n-2}{n}} \\
& \leqq \frac{c_{2}}{(R-\varrho)^{2}} \int_{B_{R}}\left|\Delta_{h} u\right|^{\alpha}\left(2+\sum_{j=1}^{n}\left(\left|u_{x_{j}}(x)\right|+\left|u_{x_{j}}\left(x+h e_{s}\right)\right|\right)^{q_{j}-2}\right) d x \\
& \leqq \frac{c_{2}}{(R-\varrho)^{2}} \int_{B_{R}}\left|\Delta_{h} u\right|^{\alpha}\left(c_{3}+\sum_{j=1}^{s}\left(\left|u_{x_{j}}(x)\right|+\left|u_{x_{j}}\left(x+h e_{s}\right)\right|\right)^{q_{j}-2}\right) d x \\
& \leqq \frac{c_{4}}{(R-\varrho)^{2}} \sum_{j=1}^{s}\left(\int_{B_{R}}\left(1+\left|u_{x_{j}}(x)\right|+\left|u_{x_{j}}\left(x+h e_{s}\right)\right|\right)^{q_{j}} d x\right)^{\frac{q_{j}-2}{2}}\left(\int_{B_{R}}\left|\Delta_{h} u\right|^{\frac{\alpha q_{j}}{2}} d x\right)^{\frac{2}{q_{j}}}
\end{aligned}
$$


Since $u_{x_{j}} \in L^{q_{j}}(\Omega)$ and since $q_{j} \leqq q_{s}$ if $j \leqq s$, we can bound the right hand side by

$$
\frac{c_{5}}{(R-\varrho)^{2}}\left(\int_{B_{R}}\left|\Delta_{h} u\right|^{\alpha q_{s}} \frac{2}{2} d x\right)^{\frac{2}{q_{s}}}
$$

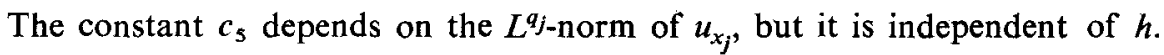
We can go to the limit as $h \rightarrow 0$, by using a standard argument (see for example [7], Section 7.11, or [8], Section III-3, or [2], Proposition IX.3), and we obtain (4.2).

Lemma 4.2. Let us assume that (1.6), (1.7), (4.1) and (3.8) hold. Let us assume also that $u_{x_{s+1}}, u_{x_{s+2}}, \ldots, u_{x_{n}} \in L_{\mathrm{loc}}^{\infty}(\Omega)$. Let $R_{1}$ be a fixed radius such that $B_{R_{1}} \subset \subset \Omega$. Then there is a constant $c_{6}$ such that

$$
\left\|u_{x_{s}}\right\|_{L^{\infty}\left(B_{R_{1} ! 2}\right)} \leqq c_{6}\left\|u_{x_{s}}\right\|_{L^{q_{S}\left(B_{R_{1}}\right)}}
$$

Proof. Let us take $R, \varrho \in\left(R_{1} / 2, R_{1}\right]$ such that

$$
R=R_{k}, \quad \varrho=R_{k+1}, \quad \text { where } R_{k}=R_{1}\left(\frac{1}{2}+\frac{1}{2^{k}}\right), \quad k \in \mathbb{N} .
$$

We have $R-\varrho=R_{k}-R_{k+1}=R_{1} 2^{-(k+1)}$. Let us define by induction a sequence $\alpha_{k}$ as follows: let $\alpha_{1}=2$ and let

$$
\alpha_{k+1}=\frac{2 n \alpha_{k}}{q_{s}(n-2)}
$$

(if $n=2$ we replace the symbol $n /(n-2)$ with a real number $p$ large enough that (4.8) is satisfied). It is easy to see that

$$
\alpha_{k}=2 \lambda^{k-1} \quad \text { where } \lambda=\frac{2 n}{q_{s}(n-2)} .
$$

Since $q_{s}<2 n /(n-2)$, then

$$
\lambda=\frac{2 n}{q_{s}(n-2)}>1 .
$$

With these notations, by (4.2) we have

$$
\left(\int_{B_{R_{k+1}}}\left|u_{x_{s}}\right|^{\frac{\alpha_{k}}{n-2}}\right)^{\frac{1}{\lambda}} \leqq\left(\frac{c_{1} 4^{k+1}}{R_{1}^{2}}\right)^{\frac{q_{s}}{2}} \int_{B_{R_{k}}}\left|u_{x_{s}}\right|^{\frac{\alpha_{k} q_{s}}{2}} d x
$$

Let us define

$$
A_{k}=\left(\int_{B_{R_{k}}}\left|u_{x_{s}}\right|^{\frac{\alpha_{k} q_{s}}{2}} d x\right)^{\frac{1}{\lambda^{k-1}}}=\left(\int_{B_{R_{k}}}\left(\left|u_{x_{s}}\right|^{\frac{q_{s}}{2}}\right)^{\alpha_{k}} d x\right)^{\frac{2}{\alpha_{k}}}
$$


By (4.6) and (4.9) we deduce

$$
A_{k+1} \leqq\left(\frac{c_{1} 4^{k+1}}{R_{1}^{2}}\right) \frac{q_{s}}{2 \lambda^{k-1}} \cdot A_{k}
$$

Then, by induction, we obtain

$$
A_{k+1} \leqq \prod_{i=1}^{k}\left(\frac{c_{1} 4^{i+1}}{R_{1}^{2}}\right)^{\frac{q_{s}}{2 \lambda^{i-1}}} \cdot A_{1}
$$

Let us show that the product is bounded:

$$
\begin{gathered}
\prod_{i=1}^{k}\left(\frac{c_{1} 4^{i+1}}{R_{1}^{2}}\right)^{\frac{q_{s}}{2 \lambda^{i-1}}}=e^{\frac{q_{s}}{2} \sum_{i=1}^{k} \frac{1}{\lambda i-1} \log \left(\frac{c_{1} 4^{i+1}}{R_{1}^{2}}\right)} \\
\leqq e^{c_{7} \sum_{i=1}^{\infty} \frac{i+1}{\lambda^{i-1}}}=c_{8}<+\infty
\end{gathered}
$$

Thus $A_{k} \leqq c_{8} A_{1}$. Now we go to the limit as $k \rightarrow+\infty$. Since $\alpha_{k} \rightarrow+\infty$ (by (4.7) and (4.8)), by the representation of $A_{k}$ in the right-hand side of (4.10) we get

$$
\begin{aligned}
\left\|\left|u_{x_{S}}\right|^{\frac{q_{s}}{2}}\right\|_{L^{\infty}\left(B_{R_{1} / 2}\right)}^{2} & =\lim _{k \rightarrow+\infty}\left\{\left(\int_{B_{R_{1} / 2}}\left(\left|u_{x_{s}}\right|^{\frac{q_{s}}{2}}\right)^{\alpha_{k}} d x\right)^{\frac{1}{\alpha_{k}}}\right\}^{2} \\
& \leqq \limsup _{k \rightarrow+\infty} A_{k} \leqq c_{8} A_{1}=c_{8} \int_{B_{R_{1}}}\left|u_{x_{s}}\right|^{q_{s}} d x .
\end{aligned}
$$

This implies (4.5) with $c_{6}=c_{8}^{1 / q_{s}}$.

Now we are ready to complete the proof of Theorem $A$. In fact it is sufficient to apply Lemma $4.2 n$ times by choosing $s$ in the order $s=n, n-1, \ldots, 2,1$.

\section{Proof of Theorem B}

In this section we assume that $f$ is a function of class $C^{2}\left(\mathbb{R}^{n}\right)$ satisfying (1.9) and (1.10). By (1.9) there are positive constants $m_{1}, M_{1}$ and $c_{1}$ such that

$$
m_{1}|\xi|^{2}-c_{1} \leqq f(\xi) \leqq M_{1}\left(1+|\xi|^{q}\right) \quad \forall \xi \in \mathbb{R}^{n}
$$

Since we assume that $u$ is a minimizer of the integral (1.1) in the Sobolev space $H_{\text {loc }}^{1, q}(\Omega)$, we have the same situation as in the preceding section, with $q_{j}=q$ for every $j=1,2, \ldots, n$. Then the proof of Theorem B can be obtained in the same way as in the proof of Theorem $A$.

However, in this case, it is possible to proceed more directly by mean of the following Lemma.

Lemma 5.1. Let $f$ be a function satisfying (1.9) and let $u \in H_{\mathrm{loc}}^{1, q}(\Omega)$. be a minimizer of the integral (1.1). Then there is a constant $c_{2}$ such that, for $B_{Q} \subset B_{R} \subset \subset \Omega$ and for every $\alpha \geqq 2$,

$$
\sum_{s=1}^{n}\left\|u_{x_{s}}\right\|_{L^{\frac{\alpha n}{n-2}\left(B_{Q}\right)}}^{1 / \alpha} \leqq \frac{c_{2}}{(R-\varrho)^{2}}\|1+|D u|\|_{L^{q}\left(B_{R}\right)}^{q-2} \cdot \sum_{s=1}^{n}\left\|u_{x_{s}}\right\|_{L^{\frac{\alpha q}{2}\left(B_{R}\right)}}^{1 / \alpha}
$$


The proof of Lemma 5.1 is similar to the proof of Lemma 4.1; thus we do not give the details.

Then we obtain the proof of Theorem B with the method of Lemma 4.2, by using the assumption $q<2 n /(n-2)$.

\section{Proof of Theorem $\mathrm{C}$}

In this section we assume that $f$ is a function of class $C^{2}\left(\mathbb{R}^{n}\right)$ that satisfies (1.9) and (1.11).

Let us first introduce some notations. For $\alpha \in(1,2]$ let us denote by $g_{\alpha}(t)$ the function of the real variable $t$ :

$$
g_{\alpha}(t)=\left(1-t^{2} t^{2}\right)^{\frac{a-2}{2}} t
$$

Lemma 6.1. For every $\alpha \in(1,2] \mathrm{g}_{\alpha}$ is a Lipschitz-continuous function that satisfies

$$
\begin{aligned}
g_{x}^{2}(t) & \leqq \frac{1}{\alpha-1} g_{x}^{\prime}(t) \cdot\left(1+t^{2}\right)^{\alpha / 2} ; \\
g_{x}^{\prime}(t) & \geqq(\alpha-1)\left(1+t^{2}\right)^{(x-2) / 2} .
\end{aligned}
$$

The proof of Lemma 6.1 is based on computations similar to those in the proof of Lemma 3.1, and we do not give the details.

Lemma 6.2. Let $f$ satisfy (1.9) and let $u \in H_{\text {loc }}^{1, q}(\Omega)$ be a minimizer of the integral (1.1). Then there is a constant $c_{1}$ (independent of $u$ ) such that, for $B_{Q} \subset B_{R} \subset \subset \Omega$ and for $1<\alpha \leqq 2$, we have

$$
\left(\int_{B_{Q}}|D u|^{\frac{\alpha n}{n-2}} d x\right)^{\frac{n-2}{n}} \leqq \frac{c_{1}}{(R-\varrho)^{2}}\left(\frac{\alpha}{\alpha-1}\right)^{2} \int_{B_{R}}(1+|D u|)^{q-2+\alpha} d x
$$

Proof. First we proceed as in Section 3 to get a result of the type stated in Lemma 3.2. If $\eta$ is taken as in (4.3), then, as in (4.4), we obtain

$$
\begin{gathered}
\left(\int_{B_{Q}}\left|\Delta_{h} u\right|^{\frac{\alpha_{n}}{n-2}} d x\right)^{\frac{n-2}{n}} \\
\leqq \frac{c_{2}}{(R-\varrho)^{2}}\left(\frac{\alpha}{\alpha-1}\right)^{2} \int_{B_{R}}\left(1+\left|\Delta_{h} u\right|^{2}\right)^{\frac{\alpha}{2}}\left(2+|D u(x)|+\left|D u\left(x+h e_{s}\right)\right|\right)^{q-2} d x .
\end{gathered}
$$
(6.4).

Since $u \in H_{\mathrm{loc}}^{1, q}(\Omega)$ and $\alpha \leqq 2$, we can go to the limit as $h \rightarrow 0$ and we obtain

Lemma 6.3. Let $f$ satisfy (1.9), (1.11) and let $u \in H_{\mathrm{loc}}^{1, q}(\Omega)$ be a minimizer of the integral (1.1). Let $R_{1}>0$ such that $B_{R_{1}} \subset\left(\Omega\right.$. There are constants $c_{3}>0$ and $\alpha \in(1,2]$ such that, if $R_{1} / 2 \leqq \varrho<R \leqq R_{1}$, then

$$
\left(\int_{B_{0}}|D u|^{q} d x\right)^{\frac{\alpha}{q}} \leqq \frac{c_{3}}{(R-\varrho)^{2}}\left(\int_{B_{R}}(1+|D u|)^{2} d x\right)^{\frac{q-2+\alpha}{2}} .
$$


Proof. Let us show that there exists a real number $\alpha$ such that

$$
1<\alpha \leqq 2, \quad q \leqq \frac{\alpha n}{n-2}, \quad q-2+\alpha \leqq 2 .
$$

In fact these inequalities can be written in the form

$$
\alpha \in(1,2] \cap\left[q \frac{n-2}{n}, 4-q\right],
$$

and the interval in the right-hand side is not empty if and only if

$$
1<4-q, \quad q \frac{n-2}{n} \leqq 4-q \quad\left(\Rightarrow q \frac{n-2}{n} \leqq 2\right) ;
$$

that is $q<3$ and $q \leqq 2 n /(n-1)$, in accordance with assumption (1.11).

By (6.4), (6.7) and the fact that the function

$$
p \rightarrow\left(\frac{1}{|\Omega|} \int_{\Omega}|v|^{p} d x\right)^{\frac{1}{p}}
$$

is increasing with respect to $p$, we get (6.6).

The integral $(1.1)$ is well defined for $u \in C^{1}(\bar{\Omega})$. By (1.9) there exists a positive constant $c_{4}$ such that

$$
-c_{\mathbf{4}} \leqq f(\xi) \leqq c_{4}\left(1+\xi^{q}\right) \quad \forall \xi \in \mathbb{R}^{n}
$$

(more precisely (5.1) holds). Thus, by continuity, the integral $(1.1)$ is well defined in $C^{1}(\Omega)$ and in the Sobolev space $H_{\mathrm{loc}}^{1, q}(\Omega)$ too. We extend it to $H^{1,2}(\Omega)$ "by semicontinuity", using the method of [11]. Precisely, for every $u \in H^{1,2}(\Omega)$, we define

$$
F(u)=\inf \left\{\lim _{k} \inf \int_{\Omega} f\left(D u_{k}\right) d x: u_{k} \in H_{\mathrm{loc}}^{1, q}(\Omega) \cap H_{0}^{1,2}(\Omega)+u, \quad u_{k} \frac{H^{1,2}(\Omega)}{}-u\right\} .
$$

Let us notice that $F$ is an extension to $H^{1,2}(\Omega)$ of the integral defined on $H_{\mathrm{loc}}^{1, q}(\Omega)$, since the integral is lower semicontinuous in the weak topology $(\rightarrow)$ of $H^{1,2}(\Omega)$. This means that (see [11]):

$$
F(u)=\int_{\Omega} f(D u) d x \quad \forall u \in H_{\text {loc }}^{1, q}(\Omega) .
$$

Let $\Omega^{\prime}$ be an open set whose closure is contained in $\Omega$. Let $\varepsilon \in(0,1]$. Let us define a functional $F_{\varepsilon}$ by

$$
F_{\varepsilon}(u)=F(u)+\varepsilon \int_{\Omega^{\prime}}|D u|^{q} d x \quad \forall u \in H^{1,2}(\Omega) \cap H^{1, q}\left(\Omega^{\prime}\right) ;
$$

moreover we define $F_{\varepsilon}(u)=+\infty$ if $u \in H^{1,2}(\Omega)$ but $u \notin H^{1, q}\left(\Omega^{\prime}\right)$.

Lemma 6.4. Let $u$ be a minimizer on $H^{1,2}(\Omega)$ of $F(u)$. Let $u_{\varepsilon}$ be a minimizer of $F_{\varepsilon}(u)$ in the Sobolev class $H_{0}^{1,2}(\Omega)+u$. Then, as $\varepsilon \rightarrow 0, u_{\varepsilon}$ converges to u strongly in $H^{1,2}(\Omega)$. 
Proof. Let us first prove that $u_{\varepsilon}$ converges to $u$ in the weak topology of $H^{1,2}(\Omega)$. By (5.1) $u_{\varepsilon}$ is bounded in $H^{1,2} \Omega$ ) independently of $\varepsilon$. Let $u_{\varepsilon_{k}}$ be a sequence that weakly converges in $H^{1,2}(\Omega)$ to a function $u_{0} \in H^{1,2}(\Omega)$. We will prove that $u_{0}=u$.

By the same definition (6.9) $F$ is lower semicontinuous in the weak topology of $H^{1,2}(\Omega)$. Since $u_{\varepsilon_{k}}$ minimizes $F_{\varepsilon_{k}}$, for every $v \in H_{0}^{1,2}(\Omega)+u$ we obtain

$$
F\left(u_{0}\right) \leqq \liminf _{k \rightarrow+\infty} F\left(u_{\varepsilon_{k}}\right) \leqq \liminf _{k \rightarrow+\infty} F_{\varepsilon_{k}}\left(u_{\varepsilon_{k}}\right) \leqq \lim _{k \rightarrow+\infty} F_{\varepsilon_{k}}(v) \text {. }
$$

Now we consider $v \in\left(H_{0}^{1,2}(\Omega)+u\right) \cap H^{1, q}\left(\Omega^{\prime}\right)$. For such functions $v$ we have $F_{\varepsilon}(v) \rightarrow F(v)$ as $\varepsilon \rightarrow 0$. Thus we obtain

$$
F\left(u_{0}\right) \leqq F(v) \quad \forall v \in\left(H_{0}^{1,2}(\Omega)+u\right) \cap H^{1, q}\left(\Omega^{\prime}\right) .
$$

By the definition (6.9) of $F$, for every $v \in H^{1,2}(\Omega)$ there exists a sequence $v_{k} \in\left(H_{0}^{1,2}(\Omega)+u\right) \cap H_{\mathrm{loc}}^{1, q}(\Omega)$ such that $F\left(v_{k}\right)$ converges to $F(v)$ as $k \rightarrow+\infty$. By (6.12) we obtain

$$
F\left(u_{0}\right) \leqq \lim _{k \rightarrow+\infty} F\left(v_{k}\right)=F(v) .
$$

Thus $u_{0}$ is a minimizer for $F$ in $H_{0}^{1,2}(\Omega)+u$.

By the left side of (1.9) and by Taylor's formula we have

$$
f(\eta) \geqq f(\xi)+\sum_{i=1}^{n} f_{\xi_{i}}(\xi)\left(\eta_{i}-\xi_{i}\right)+\frac{m}{2}|\eta-\xi|^{2} .
$$

Let $v, w \in H_{\text {loc }}^{1, q}(\Omega)$. We put $\xi=(D v+D w) / 2$; we put also first $\eta=D v$, then $\eta=D w$, and we add the two relations. We integrate on $\Omega$ and we obtain

$$
F\left(\frac{v+w}{2}\right)+\frac{m}{2} \int_{\Omega}|D(v-w)|^{2} d x \leqq \frac{1}{2}[F(v)+F(w)] .
$$

Now if $v, w \in H^{1,2}(\Omega)$, then we take $v_{k}, w_{k} \in H_{\text {loc }}^{1, q}(\Omega)$ that converge respectively to $v, w$ in the weak topology of $H^{1,2}(\Omega)$ and satisfy

$$
F\left(v_{k}\right) \rightarrow F(v), F\left(w_{k}\right) \rightarrow F(w) .
$$

We write (6.13) for $v_{k}$ and $w_{k}$ and we go to the limit as $k \rightarrow+\infty$. By (6.14) and by the weak lower semicontinuity of $F$ and of the $H^{1,2}$-norm, we obtain (6.13) for every $v, w \in H^{1,2}(\Omega)$.

Thus the functional $F$ is strictly convex on $H_{0}^{\mathrm{I}, 2}(\Omega)+u$ and $u=u_{0}$.

Now let us prove that $F_{\varepsilon}\left(u_{\varepsilon}\right)$ converges to $F(u)$ as $\varepsilon \rightarrow 0$. Since $u_{\varepsilon}$ is a minimizer for $F_{\varepsilon}$, we have $F_{\varepsilon}\left(u_{\varepsilon}\right) \leqq F_{\varepsilon}(v)$, for every $v \in H_{0}^{1,2}(\Omega)+u$. If $v \in\left(H_{0}^{1,2}(\Omega)+u\right)$ $\cap H^{1, q}\left(\Omega^{\prime}\right)$ we obtain

$$
\limsup _{\varepsilon \rightarrow 0} F_{\varepsilon}\left(u_{\varepsilon}\right) \leqq \lim _{\varepsilon \rightarrow 0} F_{\varepsilon}(v)=F(v) .
$$

By the definition of $F$, the above inequality holds for every $v \in H_{0}^{1,2}(\Omega)+u$; in particular it holds for $v=u$. Since $F_{\varepsilon}\left(u_{\varepsilon}\right) \geqq F\left(u_{\varepsilon}\right) \geqq F(u)$. finally we obtain

$$
\lim _{\varepsilon \rightarrow 0} F_{\varepsilon}\left(u_{\varepsilon}\right)=\lim _{\varepsilon \rightarrow 0} F\left(u_{\varepsilon}\right)=F(u) .
$$


Let us use (6.13) with $v=u$ and $w=u_{\varepsilon}$ :

$$
F\left(\frac{u+u_{\varepsilon}}{2}\right)+\frac{m}{2} \int_{\Omega}\left|D\left(u-u_{\varepsilon}\right)\right|^{2} d x \leqq \frac{1}{2}\left[F(u)+F\left(u_{\varepsilon}\right)\right] .
$$

We go to the limit as $\varepsilon \rightarrow 0$. Since $\left(u+u_{\varepsilon}\right) / 2$ weakly converges to $u$, by the lower semicontinuity of $F$ and by (6.15), we obtain

$$
F(u)+\frac{m}{2} \lim \sup _{\varepsilon \rightarrow 0} \int_{\Omega}\left|D\left(u-u_{\varepsilon}\right)\right|^{2} d x \leqq F(u) .
$$

Since $F(u)<+\infty$, as $\varepsilon \rightarrow 0, u_{\varepsilon}$ converges to $u$ strongly in $H^{1,2}(\Omega)$. Lemma 6.4 is now proved.

We are ready to conclude the proof of Theorem $\mathrm{C}$. The function $u_{\varepsilon}$ is a minimizer for $F_{\varepsilon}$ on $H_{0}^{1,2}(\Omega)+u$. By the definition of $F_{\varepsilon}, u_{\varepsilon}$ is also a minimizer of $F_{\varepsilon}$ on $H^{1, q}\left(\Omega^{\prime}\right)$, when the integral if restricted to $\Omega^{\prime}$. The integrand $f_{\varepsilon}$, defined by

$$
f_{\varepsilon}(\xi)=f(\xi)+\varepsilon|\xi|^{q},
$$

is a function of class $C^{2}\left(\mathbb{R}^{n}\right)$ satisfying (1.9), (1.11) with constants $m$ and $M^{\prime}=$ $M^{\prime}(q)$ independent of $\varepsilon \in(0,1]$. By Lemma 6.3 , if $B_{R} \subset \subset \Omega^{\prime}$, we have

$$
\left(\int_{B_{Q}}\left|D u_{\varepsilon}\right|^{q} d x\right)^{\frac{\alpha}{q}} \leqq \frac{c_{3}}{(R-\varrho)^{2}}\left(\int_{B_{R}}\left(1+\left|D u_{\varepsilon}\right|\right)^{2} d x\right)^{\frac{q-2+\alpha}{2}} .
$$

Now we go to the limit as $\varepsilon \rightarrow 0$. By Lemma $6.4 u_{\varepsilon}$ converges to $u$ in the norm of $H^{1,2}(\Omega)$. Thus the right-hand side is continuous with respect to $u_{\varepsilon}$, while the lefthand side is lower semicontinuous. As $\varepsilon \rightarrow 0$ we obtain the inequality stated in Theorem C.

\section{Some other results}

First let us briefly recall how to use the $H^{1, \infty}$-regularity of the minimizers to get higher regularity.

Theorem D. Let the assumptions (1.6), (1.7) and (1.8) of Theorem $A$ or the assumptions (1.9) and (1.11) of Theorem $C$ be satisfied. If the derivatives of $f$ are Holder continuous with exponent $\alpha \in(0,1)$ up to the order $k \geqq 2$, then every minimizer $u$ of the integral (1.1) is of class $C_{\mathrm{loc}}^{k, \alpha}(\Omega)$. In particular, if $f \in C^{\infty}\left(\mathbb{R}^{n}\right)$ then $u \in C^{\infty}(\Omega)$.

Sketch of proof. By either Theorem A or Theorem C, $u \in H_{\text {loc }}^{1, \infty}(\Omega)$. Since $f \in C^{2}\left(\mathbb{R}^{n}\right)$, for every $\Omega^{\prime} \subset \subset \Omega$ there is a constant $c_{1}$ such that

$$
m|\lambda|^{2} \leqq \sum_{i j} f_{\xi_{i} \xi_{j}}(D u(x)) \lambda_{i} \lambda_{j} \leqq c_{1}|\lambda|^{2} \quad \forall \lambda \in \mathbb{R}^{n},
$$

for almost every $x \in \Omega^{\prime}$. Now the proof is standard, for example as in Section 6 of Chapter 4 of LADYZHENSKAYA \& URAL'TSEVA [9] (see also Section 1.11 by Mor- 
REY [13] and Section 8 of Chapter V by GIUSTI [8]): First, by using the difference quotient as in Section 3, for every $s \in\{1,2, \ldots, n\}$ we obtain

where

$$
\int_{\Omega^{\prime}} \sum_{i j} a_{i j}(x)\left(u_{x_{s}}\right)_{x_{j}} \varphi_{x_{i}} d x=0 \quad \forall \varphi \in C_{0}^{1}\left(\Omega^{\prime}\right)
$$

$$
a_{i j}(x)=f_{\xi_{i} \xi_{j}}(D u(x)) .
$$

Thus the partial derivative $u_{x_{s}}$ is a weak solution of a second order linear elliptic equation with bounded coefficients in $\Omega^{\prime}$. By the DE GIORGI-NASH theorem [3] $u_{x_{s}}$ is Hölder continuous in $\Omega^{\prime}$. Thus the coefficients $a_{i j}(x)$ are Hölder continuous. This implies (see, for example, Giaquinta [4], Theorem 3.2 of Chapter 3 ) that $u_{x_{s}} \in C_{\mathrm{loc}}^{1, \beta}\left(\Omega^{\prime}\right)$ for some $\beta \in(0,1)$. Then $a_{i j}(x) \in C_{\mathrm{loc}}^{\mathbf{0 , \alpha}}\left(\Omega^{\prime}\right)$; finally it follows that $u \in C_{\text {loc }}^{2, \alpha}\left(\Omega^{\prime}\right)$. The proof is complete if $k=2$. Higher regularity can be obtained by induction.

Let us show that integrals of the type considered in this paper may have discontinuous minimizers if the exponent $q$ is large in dependence of $n$. The example that we propose can be found in [5] and [12].

Let $n>3$ and let us consider the integral

$$
\int_{\Omega}\left\{\frac{1}{2} \sum_{i=1}^{n-1} u_{x_{i}}^{2}+\frac{1}{q}\left|u_{x_{n}}\right|^{q}\right\} d x
$$

where $x \equiv\left(x_{i}\right), i=1,2, \ldots, n$, and

$$
q>2 \frac{n-1}{n-3}
$$

Let $\Omega \subset\left\{x \in \mathbb{R}^{n}: x_{n}>0\right\}$. Then a minimizer of the integral $(7.1)$ is given by

$$
u\left(x_{1}, x_{2}, \ldots, x_{n}\right)=\left(c x_{n}^{q} / \sum_{i=1}^{n-1} x_{i}^{2}\right)^{\frac{1}{q-2}}
$$

where

$$
c=\left(\frac{n-1}{q-1}-\frac{2}{q-2}\right) \cdot\left(\frac{q-2}{q}\right)^{q-1} .
$$

The constant $c$ is positive and the function $u$ is unbounded near the line $x_{1}=x_{2}=\ldots=x_{n-1}=0$. The discontinuity on a line is not an accident; in fact a minimizer cannot have discontinuities in the interior of a ball, for example at a single point.

We can modify the previous example by a little considering (1.1) on a bounded set $\Omega \subset\left\{x \in \mathbb{R}^{n}: x_{n}>c_{2}\right\}$, with $c_{2}>0$, and with $f$ satisfying

$$
f(\xi)=\frac{1}{2} \sum_{i=1}^{n-1} \xi_{1}^{2}+g\left(\xi_{n}\right),
$$

where, for some positive constants $c_{3}$ and $c_{4}$,

$$
g \in C^{\infty}(\mathbb{R}), \quad g^{\prime \prime} \geqq c_{3}, \quad g(t)=\frac{1}{q} t^{q} \quad \text { if }|t| \geqq c_{4} .
$$


This integrand satisfies all the assumptions of Theorems A, B, C if $q(\geqq 2)$ is small. Thus, if $q$ is small, every minimizer is of class $C^{\infty}(\Omega)$; while, if $(7.2)$ holds, then the function $u$ given by (7.3) is a discontinuous minimizer in the Sobolev class of functions such that

$$
u_{x_{i}} \in L^{2}(\Omega) \quad \forall i=1,2, \ldots, n-1 \quad u_{x_{n}} \in L^{q}(\Omega) .
$$

Finally we wish to emphasize the importance in Theorem $\mathrm{C}$ of the extension of the integral (1.1) from $H^{1, q}(\Omega)$ to $H^{1,2}(\Omega)$. From a classical point of view the integral (1.1) is well defined for every $u \in C^{1}(\Omega)$ (the integral is well defined also if $u \notin C^{1}(\bar{\Omega})$, since the integrand is bounded from below). Under the assumption (1.9) the integral can be extended by continuity to $H^{1, q}(\Omega)$ and, by semicontinuity, to $H^{1,2}(\Omega)$. Somebody could disagree with this kind of extension (and really somebody who disagrees still exists); in any case here we present a consequence of Theorem $\mathrm{C}$ that is independent of the definition of the integral outside of $C^{1}(\Omega)$ (but that has been proved by using a right extension).

Theorem E. Let $f$ be a function of class $C^{2, \alpha}\left(\mathbb{R}^{n}\right)$ satisfying (1.9) and (1.11). Let $u_{0} \in H_{0}^{1, q}(\Omega)$. Then there is a unique solution of the problem

$$
\min \left\{\int_{\Omega} f(D u) d x: u \in C^{1}(\Omega), u-u_{0} \in H_{0}^{1,2}(\Omega)\right\} .
$$

Moreover the minimizer belongs to $C_{\mathrm{loc}}^{2, \alpha}(\Omega)$.

Proof. The functional $F$ defined in (6.9) has a unique minimizer in the Sobolev class $H^{1,2}(\Omega)+u_{0}$. By Theorems $\mathrm{C}$ and $\mathrm{D}$ the minimizer is of class $C_{\mathrm{loc}}^{2, \alpha}(\Omega)$. The result follows from the fact that the functional $F$ is an extension of the integral (1.1), and thus it is equal to the integral for every $u \in C^{1}(\Omega)$.

\section{References}

1. E. ACERBi \& N. Fusco, A regularity theorem for minimizers of quasiconvex integrals, Arch. Rational Mech. Analysis 99 (1987), 261-281.

2. H. Brezis, Analyse fonctionnelle, Théorie et applications, Masson, 1983.

3. E. DE GroRgI, Sulla differenziabilità e l'analiticità delle estremali degli integrali multipli regolari, Mem. Accad. Sci. Torino, Cl. Sci. Fis. Mat. Nat. 3 (1957), 25-43.

4. M. Giaquinta, Multiple integrals in the calculus of variations and nonlinear elliptic systems, Annals of Math. Studies 105, Princeton Univ. Press, 1983.

5. M. GIAQuinTA, Growth conditions and regularity, a counterexample, Preprint Univ. Bonn, July 1987, to appear in Manuscripta Math.

6. M. Giaquinta \& E. GiUsti, On the regularity of minima of variational integrals, Acta Math. 148 (1982), 31-46.

7. D. Gilbarg, \& N. S. Trudinger, Elliptic partial differential equations of second order, Grundl. Math. Wiss. 224, Springer-Verlag, 1977.

8. E. Giustı, Equazioni ellittiche del secondo ordine, Quaderni UMI n. 6, Pitagora Ed., 1978. 
9. O. LADYZhensKaya \& N. UraL'TSEva, Linear and quasilinear elliptic equations, Math. in Science and Engineering 46, Academic Press, 1968.

10. P. Marcellini, Approximation of quasiconvex functions, and lower semicontinuity of multiple integrals, Manuscripta Math. 51 (1985), 1-28.

11. P. Marcellini, On the definition and the lower semicontinuity of certain quasiconvex integrals, Ann. Inst. Henri Poincaré, Analyse non linéaire 3 (1986), 391-409.

12. P. Marcellini, Un exemple de solution discontinue d'un problème variationnel dans le cas scalaire, Manuscript, September 1987.

13. C. B. Morrey, Multiple integrals in the calculus of variations, Grundl. Math. Wiss. 130, Springer-Verlag, 1966.

14. J. Moser, A new proof of De Giorgi's theorem concerning the regularity problem for elliptic differential equations, Communications Pure Appl. Math. 13 (1960), 457-468.

\section{Istituto Matematico "U. Dini" \\ Università di Firenze}

(Received February 17, 1988) 